\title{
Effect of increasing doses of long chain $n-3$ PUFA on circulating endothelial progenitor cells in the MARINA study: a randomised controlled trial
}

\author{
Z. Maniou ${ }^{1}$, W. L. Hall ${ }^{1}$, R. Gray ${ }^{1}$, F. Lewis ${ }^{1}$, P. T. Seed ${ }^{2}$, P. J. Chowienczyk ${ }^{3}$ and T. A. B. Sanders ${ }^{1}$ \\ ${ }^{1}$ School of Medicine, King's College London, Diabetes \& Nutritional Sciences Division, Franklin-Wilkins Building, 150 \\ Stamford Street, London SE1 9NH, UK, ${ }^{2}$ Division of Women's Health, St Thomas' Hospital, London SE1 7EH, UK and \\ ${ }^{3}$ Cardiovascular Division, St Thomas' Hospital, London SE1 7EH, UK
}

Bone marrow-derived endothelial progenitor cells (EPC) play an important role in tissue regeneration and repair. Low EPC counts have been associated with reduced endothelial function indicating a link with impaired endothelial repair ${ }^{(1)}$, and it has been reported that circulating EPC numbers predict the occurrence of cardiovascular events ${ }^{(2,3)}$.

The MARINA intervention trial (ISRCTN66664610) investigated whether low doses of long chain $n-3$ PUFA ( $n-3$ LCP), equal to consuming one, two or four portions of oily fish per week, would influence the circulating EPC numbers in healthy men and women (45-70 years). A parallel-design randomised controlled trial compared three levels $(0.45,0.9$ or $1.8 \mathrm{~g} / \mathrm{d})$ of $n-3$ LCP (EPA to DHA ratio 1.5) $v$. placebo (olive oil) taken for 12 months. Three hundred and sixty-seven participants (142 men, 225 women; mean age 55 years (SD 7); mean BMI 26 (SD 4)) were randomised to treatment. A blood sample was taken for EPC analysis by flow cytometry at baseline, 6 and 12 months. The total population of circulating EPCs was identified with staining with a FITC-pre-conjugated CD31 antibody (Beckman Coulter UK Ltd.), a PE-pre-conjugated CD34 antibody (Beckman Coulter UK Ltd.) and an APC-pre-conjugated KDR antibody (R \& D Systems). The early population of circulating EPC was also identified using a PE-pre-conjugated CD133 antibody (Miltenyi Bioter), with the CD34 and KDR antibodies. Of the initial cohort, 312 completed the study and EPC data were complete for 282 participants. Data were analysed using ANOVA adjusting for baseline levels, age, gender, BMI and ethnicity using STATA 11 software (StataCorp LP, College Station, TX 77845, USA). The mean value on treatment (6 and 12 months) was used in the analysis. Data are presented as medians (M) with interquartile ranges (IQR).

There were no dose-dependent effects of $n-3$ LCP on circulating EPC counts. Furthermore, the data did not confirm previous findings of a positive relationship between FMD and EPC numbers (data not shown).

\begin{tabular}{|c|c|c|c|c|c|c|c|c|}
\hline & \multicolumn{2}{|c|}{ Placebo $(n$ 65) } & \multicolumn{2}{|c|}{$0.45 \mathrm{~g} / \mathrm{d}(n 70)$} & \multicolumn{2}{|c|}{$0.9 \mathrm{~g} / \mathrm{d}(n 72)$} & \multicolumn{2}{|c|}{$1.8 \mathrm{~g} / \mathrm{d}(n 75)$} \\
\hline & $\mathrm{M}$ & IQR & $\mathrm{M}$ & IQR & $\mathrm{M}$ & IQR & $\mathrm{M}$ & IQR \\
\hline$\% \mathrm{KDR}^{+} / \mathrm{CD} 34^{+}$ & & & & & & & & \\
\hline Baseline & 0.19 & $0.11-0.36$ & 0.22 & $0.12-0.41$ & 0.24 & $0.11-0.36$ & 0.23 & $0.13-0.42$ \\
\hline 6 months & 0.19 & $0.10-0.43$ & 0.22 & $0.11-0.42$ & 0.12 & $0.06-0.27$ & 0.22 & $0.13-0.43$ \\
\hline $\begin{array}{l}12 \text { months } \\
\% \mathrm{KDR}^{+} / \mathrm{CD} 133^{+}\end{array}$ & 0.20 & $0.08-0.36$ & 0.23 & $0.12-0.38$ & 0.21 & $0.10-0.34$ & 0.25 & $0.14-0.45$ \\
\hline Baseline & 0.08 & $0.03-0.14$ & 0.07 & $0.03-0.17$ & 0.09 & $0.04-0.17$ & 0.08 & $0.04-0.12$ \\
\hline 6 months & 0.06 & $0.03-0.12$ & 0.09 & $0.03-0.17$ & 0.06 & $0.03-0.11$ & 0.08 & $0.05-0.16$ \\
\hline 12 months & 0.07 & $0.03-0.20$ & 0.11 & $0.05-0.23$ & 0.10 & $0.06-0.26$ & 0.14 & $0.05-0.27$ \\
\hline
\end{tabular}

In conclusion, the results of the MARINA study suggest that consumption of $n-3$ LCP, at levels of intake achievable from the diet, does not influence circulating EPC counts, a marker of endothelial repair capacity.

Funding for this work was provided by the Food Standards Agency and the Department of Health, England (Project code N02041).

1. Murphy C, Kanaganayagam GS, Jiang B et al. (2007) Arterioscler Thromb Vasc Biol 27, 936-942.

2. Hill JM, Zalos G, Halcox JPJ et al. (2003) N Engl J Med 348, 593-600.

3. Werner N, Kosiol S, Schiegl T et al. (2005) N Engl J Med 353, 999-1007. 